\title{
Single-cell Analysis of Mammalian Circadian Pacemaker
}

$\mathrm{A}^{\mathrm{r}}$ recent study published in Nalure Neuroscience revealed the spatiotemporal gene expression pattern in mammalian circadian pacemaker. Suprachiasmatic Nucleus (SCN). at single-cell resolution. This work was performed by researchers from Dr: YAN Jun's Lab at the Center for Excellence in Brain Science and Intelligence Technology (CEBSIT), Institute of Neuroscience. State Key Laboratory of Neuroscience. Chinese Academy of Sciences.

Circadian rhythm is a $2+$-hour time-keeping mechanism present in almost all organisms on earth. It is important for the regulation of many physiological processes such as locomotion, sleep, metabolism and so on. SCN has been considered as the "master pacemaker" of circadian rhythms in mammals. This small nucleus receives light input, generates selfsustained circadian rhythms, and synchronizes daily rhythms for the entire body. At the molecular level, the mammalian circadian clock consists of transcriptiontranslation feedback loops (TTFLs) of core clock genes.

Previous studies showed that circadian clock gene expression of SCN neurons oscillates synchronously. with topographically distributed cell groups oscillating at different phases. Many neuropeptides are known to be expressed in the SCN and neuropeptide-mediated intercellular coupling among SCN cells is believed to be crucial for the SCN to generate and maintain synchronized oscillations. In the past. the studies of SCN have focused on the cells expressing two neuropeptides: VIP and IVP. But it is unknown what the full spectrum of SCN neuron subtypes is. how these subtypes distribute within the SCN. and how the neuron subtypes within SCN function in circadian rhỵthm.

In the present study, they systematically classified SCN cell types and neuronal subtypes through singlecell RNA-sequencing (RNI-seq) using the Drop-seq technology. They further profiled circadian and lightinduced gene expression of these cell types.
First, they found that, in addition to neurons. nonneuronal cells such as ependymal cells and astrocytes also exhibit robust genome-wide circadian gene expression indicating that all cells in SCN may have celltype specific circadian functions. Interestingly, the core clock genes in all non-neuronal cells oscillate at delayed phases compared to those in neurons. Among neurons. SCN neurons showed elevated expression levels of core clock genes relative to non-SCN neurons. Furthermore. they defined five SCN neuron subtypes by the specific combination of expressed genes, named as $\mathrm{Acp}^{+} / \mathrm{Nms}^{+}$. $\mathrm{Grp}^{+} / \mathrm{Iip}^{+}, \mathrm{Iip}^{+} / \mathrm{Nms}^{+} . \mathrm{Cck}^{+} / \mathrm{Clql}^{+}$and $\mathrm{Cck}^{+} / \mathrm{Bdnf^{+ }}$ (Figure A-B). In particular. $\mathrm{Alp}^{+} / \mathrm{Nms}^{+}$. $\mathrm{Iip}^{+} / \mathrm{Nms}^{+}$ and $\mathrm{CCk}^{+} / \mathrm{Clql} 3^{+}$subtypes show robust circadian gene expression in the SCN, whereas $\mathrm{Crp}^{+} / \mathrm{Cip}^{+}$and $\mathrm{CCk}^{+} /$ $B d n f^{+}$subtypes show weak circadian gene expression (Figure C). Importantly, they found that the circadian phases of core clock genes in the Avp ${ }^{+} / \mathrm{Nms}^{+}$subtype in the dorsal SCN and the $\mathrm{Vip}^{+} / \mathrm{Nms}^{+}$subtype in the ventral $\mathrm{SCN}$ are earlier than the $\mathrm{Cck}^{+} / \mathrm{Clql}^{+}$subtype in the anterior SCN. Meanwhile, they found that SCN neuron subtypes showed different light responsiveness in that $\operatorname{Grp}^{+} /$Iip $^{+}$neurons are the most light responsive neuron subtype while $\mathrm{Cck}^{+} / \mathrm{Clql} \cdot 3^{+}$and $\mathrm{Cck}^{+} / \mathrm{Bdnf} f^{+}$ are the least light-responsive subtypes in SCN. This suggests that there is a subdivision of functions such as the generation of circadian rhythm and light response among $\mathrm{SCN}$ neuron subtypes. Imaging the whole- $\mathrm{SC}$. after tissue clearance, they obtained the complete threedimensional (3D) spatial distributions of these identified SCN neuronal subtypes (Figure D). Finally, combining laser-captured microdissection (LCM) and RNL-seq. they revealed the spatial gradients of gene expression within SCN further supporting our subdivision of SCN and providing evidences for the functional heterogeneity within SCN (Figure E).

In this work, they used advanced single-cell technologies to systematically classify SCN cell types 

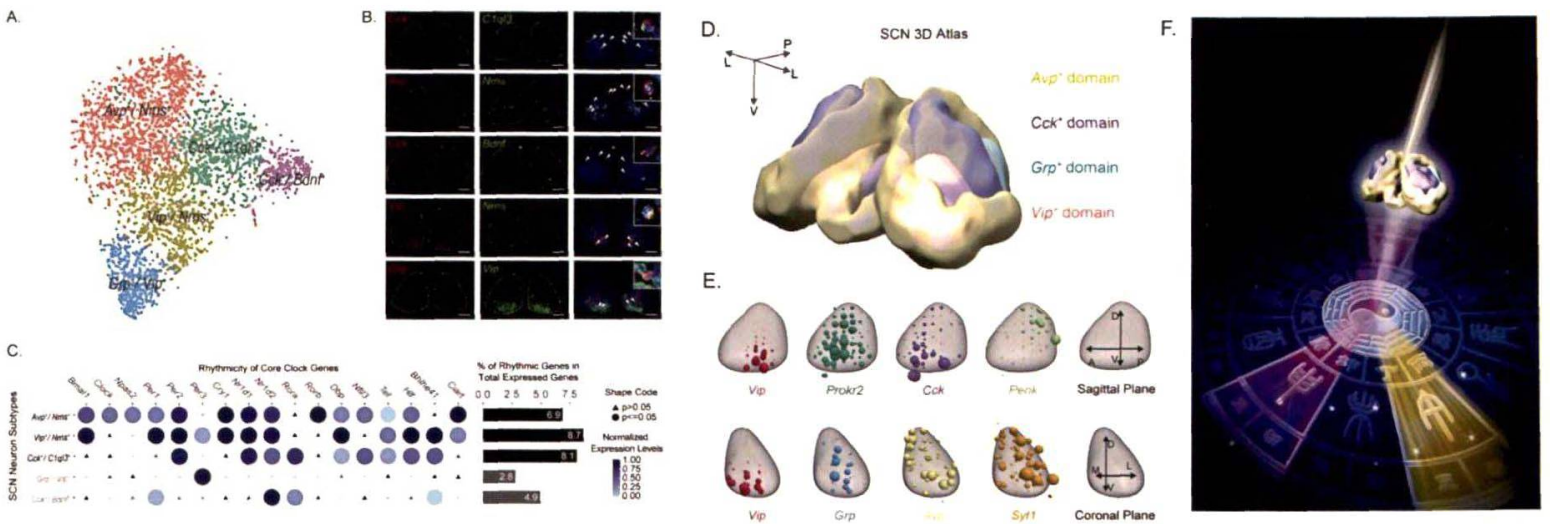

Figure legend: (A) Five SCN neuron subtypes from Drop-seq (B) SMFISH showing the co-labeling of SCN neuron markers (C) Genome-wide circadian gene expression in five SCN neuron subtypes. (D) Reconstruction of spatial domains of SCN neuron subtypes. (E) Spatial gene expression gradients revealed by LCM-seq (F) This image depicts SCN as a prism to transform the sun light into the color spectrum on the ancient Chinese sundial ( $日$ 景). This is a metaphor for the function of SCN and different circadian phases of SCN neurons. (Image by CEBSIT)

and neuronal subtypes and reconstruct their 3D spatial domains for the first time. These results provide a basis for mechanistic understanding of the structural and functional subdivision of the mammalian circadian pacemaker. Figure F depicts SCN as a prism to transform the sun light into the color spectrum on the ancient Chinese sundial. This is a metaphor for the function of $S C X N$ and different circadian phases of SCXN neurons. In humans. the disruption of circadian clock can lead to conditions such as sleep disorder and mental illness. Therefore, the understanding of neuronal mechanism of circadian rhythm in nervous system is important for the human health.

\section{Contact}

Dr. YAN Jun

Center for Excellence in Brain Science and Intelligence Technology, Chinese Academy of Sciences.

E-mail: junyan@ion.ac.cn

\section{Reference}

S. Wen, D. Ma, M. Zhao, L. Xie, Q. Wu, L. Gou, C. Zhu, Y. Fan, H. Wang, J. Yan*, (2020) Spatiotemporal single-cell analysis of gene expression in the mouse suprachiasmatic nucleus. Nature Neuroscience 23, 456. doi: 10.1038/s41593-020-0586-x. 